ISSN 0103-9954

\title{
ANÁLISE DE VIABILIDADE DA PRODUÇÃO DE SEMENTE E POLPA DE JATOBÁ (Hymenaea spp) NA REGIÃO SUL DE GOIÁS
}

\section{ANALYSIS OF VIABILITY OF SEED AND FLOUR PRODUCTION OF JATOBÁ (Hymenaea spp) IN THE SOUTHERN REGION OF GOIÁS STATE}

\author{
Camila Brás Costa ${ }^{1}$ Agostinho Lopes de Souza ${ }^{2}$ Débora Cristina Castellani ${ }^{3}$ Márcio Lopes da Silva ${ }^{4}$ \\ Eduardo Euclydes de Lima e Borges ${ }^{5}$
}

\section{RESUMO}

Abordou-se, neste trabalho, o manejo florestal sustentável de produtos florestais não madeireiros (PFNM) e avaliou a viabilidade técnica e econômica da produção de frutos de jatobá no sul goiano. Em uma primeira etapa foram mapeadas e georreferenciadas 931 árvores do gênero Hymenaea, nos municípios de Campestre de Goiás - GO, Nazário - GO e Trindade - GO. Em uma segunda etapa, foram estimados os coeficientes técnicos de produção e coletados dados, juntamente ao produtor, quanto aos custos na produção de semente e farinha. O custo de produção foi de $\mathrm{R} \$ 54,06 . \mathrm{kg}^{-1}$ e o preço de venda, de $\mathrm{R} \$ 67,58 . \mathrm{kg}^{-1}$. As análises de viabilidade econômica de plantios de jatobá e de implantação de uma Agroindústria Rural de Pequeno Porte (ARPP) indicaram a partir dos critérios de avaliação econômica VPL (Valor Presente Líquido), TIR (Taxa Interna de Retorno), B/C (razão benefício-custo) e VAE (Valor Equivalente Anual), que há viabilidade na utilização dos frutos em ambos os cenários, desde que as práticas de manejo e estratégias de conservação fomentem a produção sustentável, provendo recursos econômicos enquanto ocorre, simultaneamente, a conservação dos recursos genéticos do jatobá.

Palavras-chave: viabilidade econômica; manejo sustentável; Hymenaea courbaril.

\section{ABSTRACT}

This study addressed the sustainable forest management of non-timber forest products (NTFPs) and evaluated the technical and economic viability of producing fruits of jatoba in southern Goiás. In the first step it was mapped and georeferenced 931 trees in the genus Hymenaea at Campestre de Goiás, GO state, Nazario, GO state and Trindade, GO state. In a second step, it was estimated the coefficients of production and technical data collected by the producer as to the costs in the production of seed and flour. The production cost was $\mathrm{R} \$ 54,06 . \mathrm{kg}^{-1}$ and the sales price was $\mathrm{R} \$ 67,58 . \mathrm{kg}^{-1}$. The analysis of the economic viability of jatoba plantations and the implementation of a "Agroindústria Rural de Pequeno Porte (ARPP)" (Small Rural Agricultural Industry), indicated that from the economic evaluation criteria VPL (Net Present Value), TIR (Internal Return Rate), B/C (Benefit-cost analysis) and VAE (Equivalent Annual Value), it is viable to use the fruits in both scenarios as long as the management practices and conservation strategies encourage the sustainable production, providing

1 Engenheira Florestal, Msc., Doutoranda em Ciência Florestal, Universidade Federal de Viçosa, Av. Peter Henry Rolfs, s/n, Campus Universitário, CEP 36570-000, Viçosa (MG), Brasil. mila_ufv@yahoo.com

2 Engenheiro Florestal, Dr., Professor do Departamento de Engenharia Florestal, Universidade Federal de Viçosa, Av. Peter Henry Rolfs, s/n, Campus Universitário, CEP 36570-000, Viçosa (MG), Brasil. alsouza@ufv.br

3 Bióloga, Dra ., Gerente científico da Natura Inovação e Tecnologia de Produtos, Rod. Anhanguera, s/n, Km 30,5, Bairro Cajamar, CEP 07790-190, Cajamar (SP), Brasil. deboracastellani@natura.net

4 Engenheiro Florestal, Dr., Professor do Departamento de Engenharia Florestal, Universidade Federal de Viçosa, Av. Peter Henry Rolfs, s/n, Campus Universitário, CEP 36570-000, Viçosa (MG), Brasil.

5 Engenheiro Florestal, Dr., Professor do Departamento de Engenharia Florestal, Universidade Federal de Viçosa, Av. Peter Henry Rolfs, s/n, Campus Universitário, CEP 36570-000, Viçosa (MG), Brasil. 
economic resources while simultaneously promotes the conservation of the jatoba genetic resources. Keywords: economic viability; sustainable management; Hymeanaea courbaril.

\section{INTRODUÇÃO}

O potencial de mercado dos produtos de uso sustentável da biodiversidade vem crescendo com o aumento da variedade de produtos não tradicionais, seja em função da extração nas florestas, seja do cultivo em sistemas agroflorestais. Ao mesmo tempo, há a participação desses produtos na adição da renda dos produtores agroextrativistas em todas as regiões brasileiras e, de forma especial, nas regiões Norte, Nordeste e Centro-Oeste. No entanto, os estudos sobre os mercados para esses produtos são ainda insuficientes para atender à demanda crescente de informações que os produtores de base familiar necessitam para organizar a produção e melhorar a renda nas suas unidades produtivas (GONÇALO, 2006; FIEDLER et al., 2008).

Além disso, os produtos de origem agroextrativista não são vistos como economicamente importantes em muitos países, e por causa das dificuldades e custos na coleta de dados precisos, muitos países não coletam ou relatam seus dados. Há evidências de que em países da União Europeia - incluindo os do Mediterrâneo - os produtos não madeireiros têm importância de liderança. Em pequena escala de base florestal, empresas no Zimbábue, que, em sua maioria são baseados em produtos florestais não madeireiros, empregaram 237.000 pessoas em 1991, em comparação com 16.000 empregados no setor florestal convencional e indústrias florestais para o mesmo ano (ARNOLD et al., 1994). Na Índia, os produtos florestais não madeireiros fornecem cerca de $40 \%$ do total das receitas florestais oficiais e $55 \%$ da base florestal empregatícia (WORLD RESOURCES INSTITUTE, 1990). Pearce (1990) cita um exemplo de sucesso da economia extrativista na Indonésia, que presenciou um salto nas exportações de produtos não madeireiros de US\$ 17 milhões em 1973 para US\$ 154 milhões em 1985 e US\$ 238 milhões em 1987.

No Brasil, alguns trabalhos já verificaram a viabilidade econômica da extração sustentada de produtos não madeireiros. Santana et al. (2011) confirmaram a viabilidade econômica do extrativismo sustentável do pequi em áreas de Reserva Legal, em Iporá - GO. Bonfim (2010) abordou a utilização do pequi, baru e óleo de copaíba, além da lenha e madeira em tora, na microrregião do entorno de Brasília - DF. Quanto à exportação dos PFNM, o Ministério de Desenvolvimento do Comércio Internacional (MDCI, 2012) relata que de janeiro a novembro de 2012, o Brasil exportou, em média, cerca de US\$18 milhões de castanha-dobrasil, US\$ 207 mil de óleo essencial de eucalipto, US\$ 302 mil de palmito, US\$ 318 mil de tanino e US\$ 7,5 milhões de resina.

Quanto ao jatobá (Hymenaea spp.), são reconhecidas atualmente 14 espécies, 13 distribuídas do México à América do Sul e uma na costa leste da África, sendo descritas recentemente mais duas espécies fósseis, Hymenaea protera Poinar e Hymenaea mexicana Poinar \& Brown (LEWIS et al., 2005; POINAR JR. et al., 2002). A maioria dos táxons neotropicais tem como nomes populares mais comuns "algarrobo", "guapinol", "jatobá", "jutaî", "locust" e "copal-da-américa".

São atribuídos ao jatobá os seguintes usos da resina e polpa: combate às afecções pulmonares em geral (SHANLEY, 2005; LIMA et al., 2007; CÔRREA, 1984), dores e cólicas estomacais (LIMA et al., 2007; SHANLEY, 2005), vermífugo e antidiarreico, antioxidante, diurético, expectorante, hepatoprotetor, estimulante, energético (CÔRREA, 1984; LIMA et al., 2007), fortalecedor do sistema imunológico, estimulante utilizado para melhorar o desempenho sexual (LIMA et al., 2007); utilizado na fabricação de vernizes, (MELO et al., 2005), preparação de mingaus, farinhas, bebidas, sorvetes, picolés e licores. Já as sementes de jatobá, fontes de galactomananos e xiloglucanos, possuem características emulsificantes, espessantes, dispersantes e estabilizadoras de suspensão ou emulsão, que são de grande interesse da indústria de cosméticos, indústria farmacêutica, de alimentos (LISBOA, 2008), papel (LIMA et al, 2004), na coagulação da borracha no processo de transformação do látex (WISNIEWSKI, 1982; SHANLEY, 2005) e até como meios de cultura (LIMA-NISHIMURA et al., 2003).

Existem, no Brasil, cerca de 86.488,00 ha de plantios de jatobá, sendo que $82.811,00$ ha se encontram nos Estados de Minas Gerais e Pará, com respectivamente $78.472,00$ ha e $4.339,00$ ha (POYRY SILVICONSULT, 2011). Fundamentandose nos potenciais de utilização dos frutos de plantios 
ou de florestas naturais analisou-se, neste trabalho, a viabilidade econômica de implantação de uma agroindústria rural de pequeno porte (ARPP) para beneficiamento dos frutos na região sul de Goiás e dos plantios de jatobá.

\section{MATERIAL E MÉTODOS}

A fazenda Malícia (16 46'48's e 4943'01' W) localiza-se no município de Campestre de Goiás, GO, a $676 \mathrm{~m}$ de altitude, com área de 251,68 ha (Figura 1). O clima é tropical, com estação seca do tipo Aw pelo sistema de classificação climática de Köppen-Geiger. A temperatura média anual, segundo dados da estação meteorológica mais próxima (Anicuns - GO) é de $23,75^{\circ} \mathrm{C}$, com estação chuvosa de outubro a abril, ao passo que o período seco vai de maio a setembro. As temperaturas mais baixas são normalmente registradas entre maio e agosto. A precipitação média anual referente aos últimos cinco anos foi de 1376,65 mm (SIMEHGO, 2012). Em novembro de 2011, foram coletados por meio de entrevistas semiestruturadas, com o produtor local, os custos de coleta e beneficiamento, mão de obra e produção de frutos de Hymenaea observados na propriedade.

O potencial de produção de frutos de Hymenaea courbaril foi obtido para diferentes cenários (Tabela 1). O Cenário I corresponde à propriedade acima apresentada e foi utilizado para estruturação do custo de produção. O Cenário II corresponde às atividades de prospecção e georreferenciamento realizadas nos três municípios descritos abaixo e foi utilizado, assim como no Cenário III, para as análises de viabilidade econômica.

Os pesos médios por fruto $\left(0,04 \mathrm{~kg}\right.$. fruto $\left.^{-1}\right)$ e número de frutos por árvore (350 frutos.planta ${ }^{-1}$ ) foram obtidos em análises e medições prévias na região. Considerou-se para essas estimativas de produção, o proposto por Shanley (2005), ao considerar que as árvores de Hymenaea courbaril produzem em um ano e "descansam" no outro, multiplicando assim, a produtividade $\left(\mathrm{kg} . \mathrm{ano}^{-1}\right)$ por 0,5 . A proporção utilizada para o cálculo em $\mathrm{kg}$ de sementes e polpa a partir do peso do fruto foi de respectivamente, $1 / 7$ e 1/20, fundamentado em pesquisas bibliográficas, informações do produtor e resultados de análises parciais. Foi considerada no Cenário II a utilização de $80 \%$ da produção de frutos, atendendo ao Princípio de Precaução.

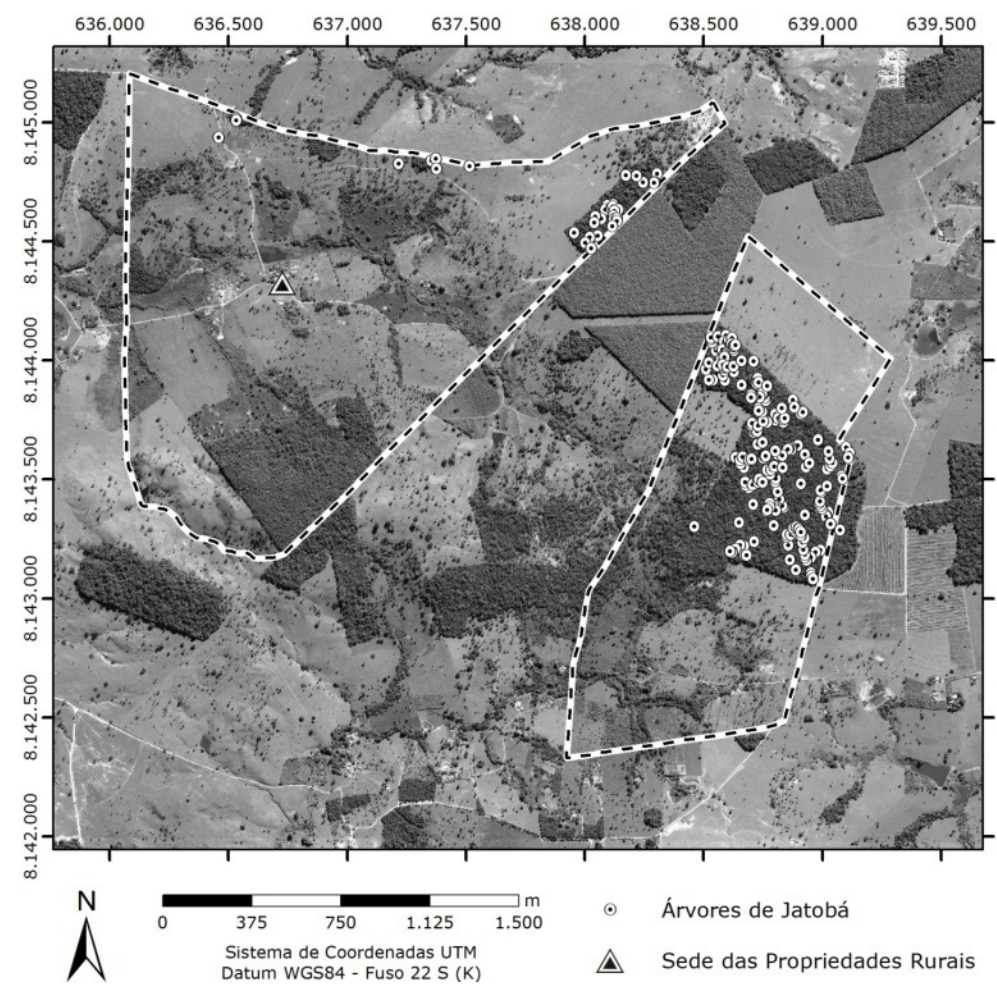

FIGURA 1: Fazenda Malícia com a área delimitada, em Campestre de Goiás, Goiás.

FIGURE 1: Farm Malícia, with enclosed highlighted area, in Campestre de Goiás, Goiás state. 
TABELA 1: Potencial produtivo anual de frutos de Hymenaea courbaril.

TABLE1: Annual potential production of fruits of Hymenaea courbaril.

\begin{tabular}{lcccccc}
\hline \multicolumn{1}{c}{ Cenários } & $\begin{array}{c}\text { N. de } \\
\text { árvores }\end{array}$ & $\begin{array}{c}\text { N. de frutos } \\
\text { por planta }\end{array}$ & $\begin{array}{c}\text { Peso por } \\
\text { fruto }(\mathrm{kg})\end{array}$ & $\begin{array}{c}\text { Produtividade } \\
(\mathrm{kg})\end{array}$ & $\begin{array}{c}\text { Semente } \\
(\mathrm{kg})\end{array}$ & $\begin{array}{c}\text { Polpa } \\
(\mathrm{kg})\end{array}$ \\
\hline $\begin{array}{l}\text { Cenário I (Fazenda do Ronan) } \\
\text { Cenário II (Nazário - GO, }\end{array}$ & 241 & & & 1687 & 241 & 84,35 \\
$\begin{array}{l}\text { Campestre de Goiás - GO e } \\
\text { Trindade - GO) }\end{array}$ & 931 & 350 & 0,04 & 6517 & 931 & 325,85 \\
\begin{tabular}{l} 
Cenário III (Plantio de 1 ha) \\
\hline
\end{tabular} & 400 & & & 5600 & 800 & 280 \\
\hline
\end{tabular}

Foram elaboradas planilhas de coeficientes técnicos de produção no software Excel incluindo insumos, mão de obra para coleta, beneficiamento e enriquecimento, equipamentos e utensílios e inventário florestal, obtendo assim as estimativas de custo na produção. Os valores dos equipamentos são relativos a janeiro de 2012, bem como salários e combustível, dentre outros. Sobre os valores dos custos de produção, foram acrescentados $10 \%$ de despesas não previstas, além do ICMS do estado de Goiás que é de 13,5\%.

No caso de insumos como energia elétrica e telefone foram considerados os valores propostos por Silva Filho (2005). No cálculo do frete levou-se em conta o número de árvores, distância das áreas de coleta em relação ao local de implantação da agroindústria, considerando 15 fretes suficientes para transporte de todos os frutos até o local idealizado para a agroindústria rural de pequeno porte. Visto que a localização da agroindústria considerou a proximidade das estradas para escoar a produção e proximidade das áreas fornecedoras, as estimativas com frete foram feitas com uma distância média de $25 \mathrm{~km}$, portanto, o valor do frete é igual ao dobro do valor do combustível $(\mathrm{R} \$ 2,05)$ vezes a distância $(\mathrm{km})$.

Segundo Silva Filho (2005), o custo de produção influencia na viabilidade econômica do projeto de implantação da agroindústria rural de pequeno porte. Além disso, o projeto deve atender também à viabilidade social, ambiental e técnica. Para atender à viabilidade social foram calculados, no lugar de dia trabalhado para as atividades de coleta dos frutos, práticas de enriquecimento e a reposição de nutriente no solo, considerou-se um colhedor com salário mínimo e com encargos sociais e trabalhistas, deixando assim de ser segurado especial do INSS. O regime de contrato foi a CLT, com os respectivos encargos: INSS $(10,7 \%)$, FGTS $(8 \%)$, provisão de $13^{\circ}(2,78 \%$ mais encargos), férias (R $\$ 82,23), \mathrm{GRRF}(\mathrm{R} \$ 2,42)$ e PCMSO (R\$18,00). Para o beneficiamento, levou-se em conta a contratação de diaristas, utilizando o valor praticado no mercado local $(\mathrm{R} \$ 45,00)$, que se mostrou superior ao proposto pela legislação própria.

Para ser ambientalmente viável, foi proposto o plantio de enriquecimento em mata, tendo como custos apenas as sementes utilizadas (3 kg), já que a mão de obra é remunerada anualmente. Sabendo que $1 \mathrm{~kg}$ de semente contém aproximadamente 300 sementes e que a taxa de germinação é de 90\% (CARPANEZZI, 1981) e mortalidade de 8,33\% (ANTEZANA, 2008), estima-se que a cada $100 \mathrm{~kg}$ de sementes coletadas serão introduzidas 742 plântulas por ano no sistema. Foi proposta também a reposição de nutrientes exportados, sendo considerada para os cálculos a eficiência de utilização dos nutrientes, através do programa Nutricalc (BARROS et al., 1995).

A depreciação foi considerada para os equipamentos, tendo o freezer vida útil de 10 anos e a infraestrutura (agroindústria) considerado 20 anos. Foi utilizada a equação 1 , em que $V$ é o valor de aquisição, $R$ o valor de sucata e $n$ a vida útil (anos):

$$
D=\frac{V-R}{n}
$$

Para a formação do preço de venda unitário (PVU), utilizou-se:

$$
P V U=\frac{C U P}{1-(C C+M L)}
$$

Em que: o custo unitário de produção (CUP) é dividido por uma unidade, subtraídos o custo de comercialização (CC) e a margem de lucro (ML). O custo de comercialização pode englobar o custo de divulgação, comissões de venda, impostos 
sobre a venda, previsões para perdas e fretes e que, neste trabalho, não foi considerado. Já a margem de lucro engloba, por exemplo, a previsão para perdas e aqui foi levada em conta uma margem de $20 \%$.

O Cenário III considerou os seguintes dados obtidos por Ferreira e Sampaio (2000): têmse 400 árvores de Jatobá $\left(25 \mathrm{~m}^{2}\right.$. árvore $\left.{ }^{-1}\right)$, com produção de frutos iniciando no $10^{\circ}$ ano e volume de madeira no ano 20 de $71,25 \mathrm{~m}^{3}$. Considerouse também neste cenário que o produtor tem interesse apenas na madeira, prática comum em algumas regiões, recebendo ao longo do período de 20 anos pela opção de manter a floresta em pé - com remuneração de acordo com a taxa SELIC (11\% a.a.). O produtor receberá também por ceder o direito de colheita, considerando que ele receba $40 \%$ do valor da venda dos frutos ao ceder o direito de colheita a um colhedor local, além do lucro pela venda da madeira no fim dos 20 anos, sendo levado em conta o valor da madeira em pé. A produtividade de frutos esperada foi de 5.600 frutos.ha- ${ }^{-1}$.

A proposta do direito de colheita pode ser utilizada também por produtores que possuem as árvores na propriedade e não demonstram interesse na colheita dos frutos. Sendo assim, o produtor pode vender o direito de colheita a colhedores, dando a estes oportunidades ao mesmo tempo em que agrega valor a um produto que ainda não tinha utilização comercial.

\section{Agroindústria Rural de Pequeno Porte (ARPP)}

No caso da agroindústria, foi considerado o prazo de pagamento da estrutura em oito anos, através de financiamentos como o PRONAF Agroindústria, uma vez que o produtor conseguirá acessar o recurso.

As análises de viabilidade de implantação da ARRP foram feitas considerando o Cenário II. A taxa de juros anual utilizada foi de $11 \%$ a.a. para o horizonte de planejamento de 20 anos da agroindústria.

O estudo da viabilidade foi elaborado tendo como produtos a farinha de jatobá, as sementes e a remuneração dos rendimentos em poupança dos créditos referentes à estocagem de carbono. Considerou-se nesse cenário a oportunidade de o produtor vender a madeira dessas árvores, com exposição do solo, sem estoque de carbono. Ao optar por não cortar as árvores e por vender os frutos, ele deve receber um valor equivalente aos rendimentos em poupança dos créditos referentes à estocagem de carbono, valor mínimo pela opção do não desmatamento. Diz-se valor mínimo, pois é considerado aqui apenas o estoque de carbono, sem levar em conta o incremento de volume e biomassa ao longo desse horizonte, sendo apenas parte dos valores tangíveis dos serviços ambientais. Também não são contabilizadas a quantificação da água ou as variáveis intangíveis como a qualidade dessa água, conservação do solo, biodiversidade e qualidade do ar.

Foi considerado para este estudo a não existência de restrições no mercado de créditos de carbono e que todo carbono estocado pode ser convertido em créditos de carbono.

$\mathrm{O}$ preço de equilíbrio (PE) e produção de equilíbrio (PrE) para a semente também foram estimados a partir das equações 3 e 4 .

$$
P E=\frac{C O T}{P R O D U C ̧ \tilde{A O}}
$$

Em que: COT corresponde ao custo operacional total $\left(\mathrm{R} \$ \cdot \mathrm{kg}^{-1}\right)$ e Produção à produtividade do período.

$$
\operatorname{Pr} E=\frac{C O T}{P R E C ̧ O \cdot \mathrm{kg}^{-1}}
$$

Em que: Preço $\left(\mathrm{R} \$ . \mathrm{kg}^{-1}\right)$ corresponde ao preço praticado no mesmo período.

Para dimensionar as operações agroindustriais necessárias na produção das sementes e a farinha, foi confeccionado um fluxograma com o balanço das atividades. Cada etapa foi descrita com base em informações do produtor que já tinha prática e vivência no beneficiamento das sementes e preparação da farinha.

\section{Indicadores ou critérios econômicos}

Para a análise financeira da produção de farinha de jatobá e sementes, foram utilizados os seguintes indicadores (NAUTIYAL, 1988; SILVA et al., 1999; REZENDE e OLIVEIRA, 2001; GRAÇA, 2000):

$$
V P L=\sum_{\mathrm{j}=0}^{\mathrm{n}} \mathrm{R}_{\mathrm{j}} \cdot(1+\mathrm{i})^{-\mathrm{j}}-\sum_{\mathrm{j}=0}^{\mathrm{n}} \mathrm{C}_{\mathrm{j}} \cdot(1+\mathrm{i})^{-\mathrm{j}}(5)
$$

Em que: VPL $=$ Valor Presente Líquido, $\mathrm{R}=$ 
receitas no período analisado, $\mathrm{C}=$ custos no período analisado, $\mathrm{i}=$ taxas de juros ou desconto $\mathrm{e} \mathrm{j}=$ número de anos.

$$
V A E=\frac{V P L . i}{\left[1-(1+i)^{-j}\right]}
$$

Em que: VAE = Valor Anual Equivalente.

$$
B / C=\frac{\sum_{j=0}^{n} R_{j} \cdot(1+i)^{-j}}{\sum_{j=0}^{n} C_{j} \cdot(1+i)^{-j}}
$$

Em que: $\mathrm{B} / \mathrm{C}=$ Relação Benefício Custo, que se refere à divisão dos benefícios atualizados descontados pela taxa de juros, pelos custos anualizados. Esse cálculo permite inferir quanto de receita é gerada por uma unidade de custo. Um resultado maior que 1 resulta em receitas maiores que custos e se menores que 1 , os custos serão maiores que receitas.

TIR: É o valor de r calculado, de modo a fazer o VLP nulo. Representa o retorno intrínseco do projeto. Se for maior que a taxa usual recebida na economia, compensa realizar o empreendimento e o reverso também é verdadeiro.

Foram feitas também pesquisas quanto aos preços de mercado praticados para os diferentes produtos do jatobá, com ênfase na semente e farinha, sendo os dados coletados entre novembro de 2011 e maio de 2012.

\section{RESULTADOS E DISCUSSÃO}

\section{Etapas do processamento}

A primeira etapa do processamento é a coleta (Figura 2) e, consecutivamente, o transporte da matéria-prima em sacos de aniagem. Após a chegada dos frutos no local de beneficiamento é feita a quebra, seleção e descarte de frutos abortados. O despolpamento é realizado utilizando-se peneira de aproximadamente 60 mesh, de pequena capacidade. A partir daí, separam-se a polpa e a semente. A polpa será passada novamente em peneira de malha menor (20 a 40 mesh), configurando uma das etapas finais na preparação da farinha. Esse material é ensacado e armazenado em câmara fria. As sementes são limpas novamente e, logo após, ensacadas e estocadas para posterior comercialização.

\section{Custos de produção da farinha e semente}

Os requerimentos técnicos e custos

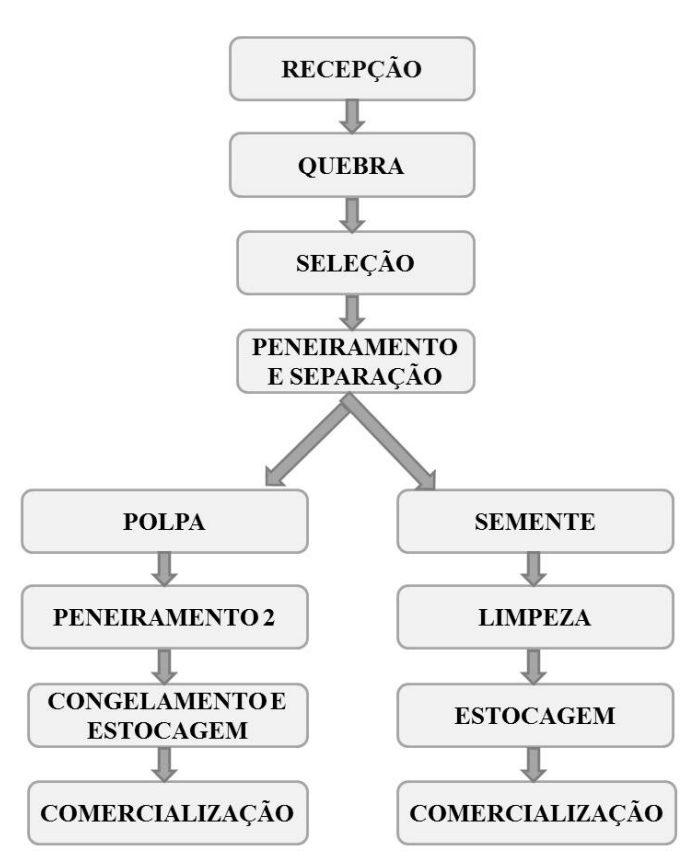

FIGURA 2: Fluxograma com as etapas envolvidas no processamento dos frutos de Jatobá.

FIGURE 2: Flowchart with steps involved in processing fruits of Jatoba.

considerados para a produção de farinha e semente de Hymenaea courbaril estão resumidos na Tabela 2. Os valores listados referem-se à produção de $100 \mathrm{~kg}$ de sementes, considerando como capacidade máxima de trabalho da agroindústria as árvores mapeadas no Cenário II $\left(750 \mathrm{~kg} . \mathrm{ano}^{-1}\right)$, ou seja, alguns dos valores serão apresentados com valor total dividido por 7,5. Foi considerada também a vida útil dos equipamentos, sendo para as caixas plásticas, por exemplo, de quatro anos. Não se levou em conta o custo da terra.

Além dos custos de produção (R\$ $4.330,38)$, foram incluídos $10 \%$ para as despesas extras e 13,5\% do ICMS que estará incluído no preço de venda, chegando a um total de $\mathrm{R} \$$ 5.406,48. Dividindo o custo total pela produção de $100 \mathrm{~kg}$ de semente obtém-se um custo unitário de produção (CUP) de R \$ 54,06. Para encontrar o preço de venda unitário (PVU), utilizou-se a equação 2 , chegando à cifra de $\mathrm{R} \$ 67,58$, com margem de lucro de $20 \%$ (equivalente a US $\$ 28,91$ ou $€ 21,33)$.

Dentre os produtos florestais não madeireiros comercializados, Balzon (2006) encontrou para o artesanato no Paraná margem de lucro para o cipó-preto (Philodendron 
TABELA 2: Custo de produção de $100 \mathrm{~kg}$ de semente e $35 \mathrm{~kg}$ de farinha na Fazenda Malícia, em Campestre de Goiás, Goiás.

TABLE 2: Production cost of $100 \mathrm{~kg}$ of seed, and $35 \mathrm{~kg}$ of flour at Farm Malícia, Campestre de Goiás, Goiás state.

\begin{tabular}{|c|c|c|c|c|}
\hline Etapas & Unidade & Quant. & $\begin{array}{c}\text { Valor } \\
\text { Unitário }(\mathrm{R} \$)\end{array}$ & $\begin{array}{l}\text { Valor Total } \\
\quad(\mathrm{R} \$)\end{array}$ \\
\hline \multicolumn{5}{|l|}{ 1. Insumos } \\
\hline Energia elétrica e telefone & Ano & 1 & 2880,00 & 384,00 \\
\hline Combustível (transporte até ARPP) & frete & 2 & 102,50 & 205,00 \\
\hline Adubação de reposição & $\mathrm{Kg}$ & 171 & 0,30 & 51,33 \\
\hline Sementes para plantio (enriquecimento) & $\mathrm{Kg}$ & 3 & 60,00 & 180,00 \\
\hline Subtotal & & & & 820,33 \\
\hline \multicolumn{5}{|l|}{ 2. Serviços } \\
\hline $\begin{array}{l}\text { Colhedor (Colheita dos frutos, plantio de mudas } \\
\text { e manutenção) }\end{array}$ & $\begin{array}{l}\text { salário } \\
\text { mínimo } \\
\text { com } \\
\text { encargos }\end{array}$ & 12 & 902,29 & 1443,66 \\
\hline Beneficiamento & dias/homem & 8 & 45,00 & 360,00 \\
\hline Inventário do recurso & unidade & 1 & 144,46 & 144,46 \\
\hline Subtotal & & & & 1948,12 \\
\hline \multicolumn{5}{|l|}{ 3. Equipamentos e infraestrutura } \\
\hline $\begin{array}{l}\text { Caixa plástica (armazenamento de polpa e } \\
\text { semente) }\end{array}$ & caixa $25 \mathrm{~L}$ & 6 & 26,00 & 5,20 \\
\hline Caixa plástica (armazenamento de polpa) & caixa $11 \mathrm{~L}$ & 3 & 17,00 & 1,70 \\
\hline Medidor de temperatura e umidade & unidade & 1 & 5,00 & 0,17 \\
\hline Etiquetas e fichas de produção & unidade & 50 & 0,50 & 25,00 \\
\hline Embalagem (armazenamento) & cento & 1 & 120,00 & 120,00 \\
\hline Peneira de 60 mesh $\left(1^{\mathrm{a}}\right.$ peneirada da polpa $)$ & unidade & 2 & 26,00 & 6,93 \\
\hline Peneira de 20 a 40 mesh ( $2^{\mathrm{a}}$ peneirada da polpa) & unidade & 2 & 26,00 & 6,93 \\
\hline EPI (agroindústria) & kit & 3 & 60,00 & 24,00 \\
\hline EPI (coletor) & kit & 1 & 49,00 & 49,00 \\
\hline Freezer horizontal 546L & unidade & 1 & 1750 & 11,67 \\
\hline Agroindústria & unidade & 1 & 40000 & 666,67 \\
\hline Subtotal & & & & 917,27 \\
\hline \multicolumn{5}{|l|}{ 4. Outros } \\
\hline Assistência técnica & dias/homem & 2 & 250,00 & 500,00 \\
\hline Depreciação & - & 1 & 1085,00 & 144,67 \\
\hline Subtotal & & & & 644,67 \\
\hline Total & & & & 4330,38 \\
\hline
\end{tabular}

melanorrhizum Reit) sem beneficiamento de $16,7 \%$; para aguaricana (Geonoma gamiova Barb Rody) detectou a mesma margem de lucro de $16,7 \%$; para a extração de sacas de musgo (Sphagnum spp) foi observado o valor de $37,5 \%$ e para as folhas de samambaia (Pteridium aquilinum), uma margem de lucro de $33,3 \%$, justificando assim os valores aqui adotados.

Quando comparado o PVU obtido àqueles praticados no mercado (Tabela 3), fornecidos por 
TABELA 3: Pesquisa de mercado sobre preços praticados de produtos oriundos do jatobá.

TABLE 3: Market research on prices of products from jatoba.

\begin{tabular}{|c|c|c|c|c|c|}
\hline Vendedor & Município & Estado & Produto & Espécie & $\begin{array}{c}\text { Preço de venda (R\$/ } \\
\text { unidade) }\end{array}$ \\
\hline $\begin{array}{l}\text { Instituto Socioambiental (ISA) - } \\
\text { Rede de Sementes do Xingu }\end{array}$ & Canarana & MT & Semente & Hymenaea sp. & $10,00 / \mathrm{kg}$ \\
\hline $\begin{array}{l}\text { Instituto Socioambiental (ISA) - } \\
\text { Rede de Sementes do Xingu }\end{array}$ & Canarana & MT & Semente & H. stigonocarpa & $10,00 / \mathrm{kg}$ \\
\hline Viveiro Flora Tietê & Penápolis & SP & Semente & H. stigonocarpa & $10,00 / \mathrm{kg}$ \\
\hline $\begin{array}{c}\text { Silvicultura UFV- Universidade } \\
\text { Federal de Viçosa }\end{array}$ & Viçosa & MG & Semente & H. courbaril & $20,00 / \mathrm{kg}$ \\
\hline Viveiro Flora Tietê & Penápolis & SP & Semente & H. courbaril & $30,00 / \mathrm{kg}$ \\
\hline Seu Domingos Foli & Linhares & ES & Semente & H. courbaril & $35,00 / \mathrm{kg}$ \\
\hline Seu Domingos Foli & Linhares & ES & Semente & H. aurea & $35,00 / \mathrm{kg}$ \\
\hline Eko Amazon & - & - & Semente & H. stigonocarpa & $35,00 / \mathrm{kg}$ \\
\hline Central das sementes & - & - & Semente & H. courbaril & $38,00 / \mathrm{kg}$ \\
\hline Arbocenter & Birigui & SP & Semente & H. stigonocarpa & $100,00 / \mathrm{kg}$ \\
\hline Arbocenter & Birigui & SP & Semente & H. courbaril & $100,00 / \mathrm{kg}$ \\
\hline Clube de sementes do Brasil & Alexânia & GO & Semente & H. courbaril & $105,00 / \mathrm{kg}$ \\
\hline $\begin{array}{l}\text { Signus Vitae - Projetos } \\
\text { Ambientais Inteligentes }\end{array}$ & $\begin{array}{l}\text { Volta } \\
\text { Redonda }\end{array}$ & RJ & Semente & H. courbaril & $110,00 / \mathrm{kg}$ \\
\hline Mata Atlântica - Viveiro Florestal & Itatiba & SP & Semente & H. courbaril & $175,00 / \mathrm{kg}$ \\
\hline Mata Atlântica - Viveiro Florestal & Itatiba & SP & Semente & H. stigonocarpa & $175,00 / \mathrm{kg}$ \\
\hline $\begin{array}{l}\text { Estabelecimento do Mercado } \\
\text { Central - Alves (2010) }\end{array}$ & $\begin{array}{l}\text { Belo } \\
\text { Horizonte }\end{array}$ & MG & $\begin{array}{l}\text { Casca da } \\
\text { árvore }\end{array}$ & Hymenaea sp. & $50,00 / \mathrm{kg}(1,50 / 30 \mathrm{~g})$ \\
\hline Estabelecimento 01 & Belém & PA & $\begin{array}{l}\text { Casca da } \\
\text { árvore }\end{array}$ & Hymenaea sp. & 2,00 (casca) \\
\hline Estabelecimento 02 & Belém & PA & $\begin{array}{l}\text { Casca da } \\
\text { árvore }\end{array}$ & Hymenaea sp. & 3,00 (casca) \\
\hline Estabelecimento 03 & Belém & PA & $\begin{array}{l}\text { Casca da } \\
\text { árvore }\end{array}$ & Hymenaea sp. & 5,00 (casca) \\
\hline $\begin{array}{l}\text { Estabelecimento do Mercado } \\
\text { Central* }\end{array}$ & $\begin{array}{c}\text { Belo } \\
\text { Horizonte }\end{array}$ & MG & Resina & Hymenaea sp. & $66,67 / \mathrm{kg}(2,00 / 30 \mathrm{~g})$ \\
\hline Estabelecimento 01 & Belém & PA & Seiva & Hymenaea sp. & $30,00 / \mathrm{L}$ \\
\hline Estabelecimento 02 & Belém & PA & Seiva & Hymenaea sp. & $20,00 / \mathrm{L}$ \\
\hline Estabelecimento 03 & Belém & PA & Seiva & Hymenaea sp. & $40,00 / \mathrm{L}(10,00 / 250 \mathrm{~mL})$ \\
\hline $\begin{array}{c}\text { Produtos Agroecológicos e } \\
\text { Extrativistas da Chapada dos } \\
\text { Veadeiros }\end{array}$ & $\begin{array}{l}\text { Alto } \\
\text { Paraíso de } \\
\text { Goiás }\end{array}$ & GO & Seiva & H. courbaril & $50,00 / \mathrm{L}(25,00 / 500 \mathrm{~mL})$ \\
\hline Central do cerrado & Diorama & GO & Farinha & Hymenaea sp. & $60,00 / \mathrm{kg}(12,00 / 200 \mathrm{~g})$ \\
\hline Saturno percussão & São Paulo & SP & $\begin{array}{l}\text { Casca do } \\
\text { fruto }\end{array}$ & Hymenaea sp. & 30,00/Molho grande \\
\hline Saturno percussão & São Paulo & SP & $\begin{array}{l}\text { Casca do } \\
\text { fruto }\end{array}$ & Hymenaea sp. & 20,00/Molho pequeno \\
\hline Amazon Produtos & Manaus & $\mathrm{AM}$ & $\begin{array}{l}\text { Xarope } \\
\text { composto }\end{array}$ & Hymenaeasp. & $89,0 / \mathrm{L}(8,9 / 100 \mathrm{~mL})$ \\
\hline
\end{tabular}


viveiristas e profissionais que constam no cadastro da Rede de Sementes do Brasil, percebe-se que o PVU proposto é justo e competitivo.

Percebe-se, portanto, que há grande variação de preços. Conforme exposto por Torres (2001), parte dessa variação para produtos florestais não madeireiros é explicada pelas diferenças na distância entre as áreas produtoras e os centros de comercialização, pela qualidade do produto, assim como pelo diferencial de informação dos produtores sobre o mercado, o que afeta sua capacidade de negociação ante os intermediários, fato esse que é favorecido pela falta de organização na produção.

A adubação de reposição indicada referese aos nutrientes exportados na coleta de frutos, multiplicada pelo número de árvores manejadas. Os resultados da análise de frutos de Campestre de Goiás, bem como os valores de macro e micronutrientes necessários à reposição por árvore seguem na Tabela 4.

Pode-se observar nesta tabela que, caso os frutos fossem quebrados em campo e transportadas apenas as sementes e a polpa, a exportação de nutrientes seria bem menor. Porém, seria mais delicada à posterior utilização da polpa para a alimentação humana, podendo até inviabilizar esse uso, uma vez que a polpa ficaria exposta até a chegada à agroindústria.

A produção de sementes e farinha, pelo perfil da atividade, apresenta-se como opção viável de exploração para a agricultura familiar.
A safra ocorre de julho a setembro, sendo mais intensa nos meses de agosto e setembro. Por corresponder a um período restrito, as famílias podem integrar esta atividade com outras de maior exigência de mão de obra, sem necessidade de recorrer à contratação de terceiros.

\section{Análise de viabilidade da implantação da agroindústria rural de pequeno porte (ARPP)}

A partir dos custos operacionais totais (COT) e das receitas totais, foram obtidos os fluxos de caixa líquido e acumulado (Tabela 5). O preço de equilíbrio (PE) e produção de equilíbrio (PrE) apresentados são referentes apenas às sementes.

OprojetoapresentouVPLdeR $\$ 660.500,06$ e $\mathrm{B} / \mathrm{C}$ maior que $1(5,18)$. Considerando-se que os custos estão diluídos ao longo do período, não houve períodos com lucro operacional negativos, impossibilitando assim o cálculo da TIR. A partir do VAE (R\$ 82942,72) e levando-se em conta os 13 proprietários cadastrados na região, pode-se considerar que a receita média por proprietário será de $\mathrm{R} \$ 531,68$.mês- ${ }^{1}$.

$\mathrm{O}$ ponto de nivelamento ou produção de equilíbrio (PrE) de semente $(966,11 \mathrm{~kg})$ em relação ao COT, indica que os $750 \mathrm{~kg}$ anuais produzidos de semente não serão suficientes para cobrir os custos operacionais totais, sendo necessário o aumento da produtividade em $216,11 \mathrm{~kg}$ ou aumento do PVU, considerando

TABELA 4: Resultados analíticos para casca, polpa e semente de Hymenaea courbaril proveniente de Campestre de Goiás e recomendação de adubação de reposição por árvore.

TABLE 4: Analytical results for epicarp, pulp and seeds of Hymenaea courbaril from Campestre de Goiás and fertilizer recommendation for tree replacement.

\begin{tabular}{|c|c|c|c|c|c|c|c|c|c|c|c|}
\hline Análise/ & $\mathrm{N}$ & $\mathrm{P}$ & $\mathrm{K}$ & $\mathrm{Ca}$ & $\mathrm{Mg}$ & $\mathrm{S}$ & $\mathrm{Zn}$ & $\mathrm{Fe}$ & $\mathrm{Mn}$ & $\mathrm{Cu}$ & B \\
\hline Parte do fruto & \multicolumn{6}{|c|}{ 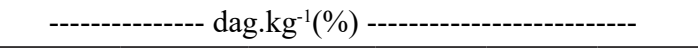 } & \multicolumn{5}{|c|}{----------------- mg.kg-1 } \\
\hline Casca & 0,572 & 0,072 & 0,651 & 0,223 & 0,061 & 0,083 & 13,1 & 71,7 & 166,3 & 0,1 & 10,7 \\
\hline Polpa & 0,958 & 0,1068 & 1,696 & 0,15 & 0,154 & 0,098 & 15,3 & 25,4 & 179,4 & 3,7 & 13 \\
\hline Semente & 1,329 & 0,1546 & 0,698 & 0,159 & 0,131 & 0,188 & 21,3 & 41,1 & 112,8 & 2 & 14,6 \\
\hline Recomendação/ & $\mathrm{N}$ & $\mathrm{P}$ & $\mathrm{K}$ & $\mathrm{Ca}$ & $\mathrm{Mg}$ & $\mathrm{S}$ & $\mathrm{Zn}$ & $\mathrm{Fe}$ & $\mathrm{Mn}$ & $\mathrm{Cu}$ & $\mathrm{B}$ \\
\hline Parte do fruto & \multicolumn{6}{|c|}{----------------------g/árvore------------------------ } & \multicolumn{5}{|c|}{-------------------------mg/árvore------------------- } \\
\hline Casca & 92,38 & 18,09 & 105,14 & 36,01 & 9,85 & 20,85 & 740,48 & 4052,84 & 9400,11 & 5,65 & 604,82 \\
\hline Polpa & 9,58 & 1,66 & 16,96 & 1,5 & 1,54 & 1,52 & 53,55 & 88,9 & 627,9 & 12,95 & 45,5 \\
\hline Semente & 37,88 & 6,85 & 19,89 & 4,53 & 3,73 & 8,33 & 212,47 & 409,97 & 1125,18 & 19,95 & 145,64 \\
\hline Total & 139,83 & 26,6 & 141,99 & 42,05 & 15,13 & 30,71 & 1006,5 & 4551,72 & 11153,19 & 38,55 & 795,95 \\
\hline
\end{tabular}


TABELA 5: Atividades envolvidas na implantação da agroindústria rural de pequeno porte para um horizonte de 20 anos e fluxos de caixa líquido e acumulado.

TABLE 5: Activities involved in the deployment of small rural agricultural industry for a 20 -year horizon and accumulated net cash flows.

\begin{tabular}{|c|c|c|c|c|c|c|c|}
\hline Anos & 1 & 2 & 3 & 4 & 5 & 6 & 7 \\
\hline \multicolumn{8}{|l|}{ Saída/ano (R\$.ano $\left.{ }^{-1}\right)$} \\
\hline 1. Insumos & 5965,1 & 5995,1 & 5995,1 & 5995,1 & 5995,1 & 5995,1 & 5995,1 \\
\hline 2. Mão de obra & 13827,5 & 13827,5 & 13827,5 & 13827,5 & 13827,5 & 13827,5 & 13827,5 \\
\hline $\begin{array}{l}\text { 3. Equipamentos e } \\
\text { infraestrutura }\end{array}$ & 6027,5 & 5482,5 & 5482,5 & 5482,5 & 6027,5 & 5482,5 & 5482,5 \\
\hline 4. Outros & 39470,4 & 12289,3 & 12289,3 & 12289,3 & 12424,7 & 12289,3 & 12289,3 \\
\hline $\begin{array}{l}\text { Custo operacional total } \\
\text { (COT) }\end{array}$ & 65290,5 & 37594,4 & 37594,4 & 37594,4 & 38274,9 & 37594,4 & 37594,4 \\
\hline \multicolumn{8}{|l|}{ Entrada/ano (R $\$$. ano $\left.^{-1}\right)$} \\
\hline Receita bruta & 66133,9 & 66133,9 & 66133,9 & 66133,9 & 66133,9 & 66133,9 & 66133,9 \\
\hline Fluxo de caixa líquido (FCL) & 843,3 & 28539,4 & 28539,4 & 28539,4 & 27859,0 & 28539,4 & 28539,4 \\
\hline Anos & 8 & 9 & 10 & 11 & 12 & 13 & 14 \\
\hline \multicolumn{8}{|l|}{ Saída/ano $\left(\mathrm{R} \$ . a^{-1}\right)$} \\
\hline 1. Insumos & 5965,1 & 5995,1 & 5995,1 & 5995,1 & 5995,1 & 5995,1 & 5995,1 \\
\hline 2. Mão de obra & 13827,5 & 13827,5 & 13827,5 & 13827,5 & 13827,5 & 13827,5 & 13827,5 \\
\hline $\begin{array}{l}\text { 3. Equipamentos e } \\
\text { infraestrutura }\end{array}$ & 5482,5 & 1027,5 & 482,5 & 482,5 & 482,5 & 1027,5 & 482,5 \\
\hline 4. Outros & 12289,3 & 11182,2 & 11046,8 & 11046,8 & 11046,8 & 11182,2 & 11046,8 \\
\hline $\begin{array}{l}\text { Custo operacional total } \\
\text { (COT) }\end{array}$ & 37594,4 & 32032,4 & 31351,9 & 31351,9 & 31351,9 & 32032,4 & 31351,9 \\
\hline \multicolumn{8}{|l|}{ Entrada/ano $\left(\mathrm{R} \$ . \mathrm{ano}^{-1}\right)$} \\
\hline Receita bruta & 66133,9 & 66133,9 & 149548,2 & 74475,3 & 232962,5 & 232962,5 & 232962,5 \\
\hline Fluxo de caixa líquido (FCL) & 28539,4 & 34101,5 & 118196,3 & 43123,4 & 201610,6 & 200930,1 & 201610,6 \\
\hline Anos & 15 & 16 & 17 & 18 & 19 & 20 & \\
\hline \multicolumn{8}{|l|}{ Saída/ano $\left(\mathrm{R} \$ . \mathrm{ano}^{-1}\right)$} \\
\hline 1. Insumos & 5995,1 & 5995,1 & 5995,1 & 5995,1 & 5995,1 & 5995,1 & \\
\hline 2. Mão de obra & 13827,5 & 13827,5 & 13827,5 & 13827,5 & 13827,5 & 13827,5 & \\
\hline $\begin{array}{l}\text { 3. Equipamentos e } \\
\text { infraestrutura }\end{array}$ & 482,5 & 482,5 & 1027,5 & 482,5 & 482,5 & 482,5 & \\
\hline 4. Outros & 11046,8 & 11046,8 & 11182,2 & 11046,8 & 11046,8 & 11182,2 & \\
\hline $\begin{array}{l}\text { Custo operacional total } \\
\text { (COT) }\end{array}$ & 31351,9 & 31351,9 & 32032,4 & 31351,9 & 31351,9 & 31351,9 & \\
\hline \multicolumn{8}{|l|}{ Entrada/ano $\left(\mathrm{R} \$ . \mathrm{ano}^{-1}\right)$} \\
\hline Receita bruta & 316376,8 & 316376,8 & 316376,8 & 399791,1 & 399791,1 & 399791,1 & \\
\hline Fluxo de caixa líquido (FCL) & 285024,9 & 285024,9 & 284344,5 & 368439,2 & 368439,2 & 368439,2 & \\
\hline
\end{tabular}

por exemplo, a contribuição de outros serviços ambientais que não foram aqui valorados. $\mathrm{O}$ preço de equilíbrio (PE) da semente de $\mathrm{R} \$ 87,05$ indica que a agroindústria rural de pequeno porte tem receita líquida negativa quando vende apenas a semente de $\mathrm{R} \$ 19,47$ a cada quilo comercializado.
Com a comercialização da farinha de jatobá, a perspectiva passa a ser diferente.

\section{Farinha do jatobá}

$\mathrm{Na}$ entrevista, o proprietário relatou não 


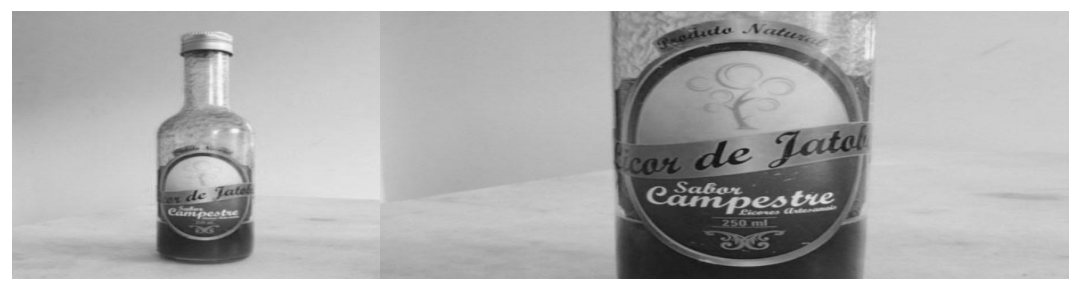

FIGURA 3: Licor de Jatobá comercializado em feiras e centros comerciais, como o aeroporto de Goiânia. FIGURA 3: Jatoba liquor sold in fairs and malls, like at the airport of Goiania.

comercializar a polpa. A alternativa encontrada para agregar valor à farinha foi no preparo de licores, que são vendidos em feiras e centros comerciais, dentre eles o aeroporto de Goiânia, no valor de R\$ 12 a garrafa de $250 \mathrm{ml}$, o equivalente a US\$5,13 ou $€ 3,79$ (Figura 3).

O proprietário já planeja apresentar a farinha de jatobá nas escolas locais com degustação de bolos, pães e "toddy" de jatobá que atendem à proposta de introdução da farinha no programa de merenda escolar ao mesmo tempo em que incentiva o desenvolvimento da produção e cultura local.

Comparando-se o preço praticado (Tabela 3) com o preço sugerido de $\mathrm{R} \$ 40 . \mathrm{kg}^{-1}$ para a farinha, percebe-se que este também foi competitivo. Os fornecedores de farinha da Central do Cerrado se encontram em Diorama - GO, na Phytobrasil (Centro de Tecnologia Agroecológica de Pequenos Agricultores) e em Nioaque - MS, na Ceppec (Centro de Produção, Pesquisa e Capacitação do Cerrado).

De acordo com Bozza (2012), os frutos nativos do Cerrado são muito nutritivos, riquíssimos principalmente em sais minerais como o potássio e zinco. As pesquisas têm aumentado nesse sentido e os resultados que surgem em publicações científicas e até mesmo na mídia em geral, trazem o jatobá como um dos frutos muito pesquisados. No entanto, mesmo diante de todos os valores nutricionais que agregam, ainda não existe um mercado formal para eles, devido principalmente à sazonalidade e às poucas iniciativas de organização para a comercialização.

\section{Análise de viabilidade econômica do plantio}

A partir dos custos totais operacionais (COT) e das receitas totais, foram obtidos os fluxos de caixa líquido e acumulado sendo apresentados na Tabela 6. O lucro operacional passa a ser positivo a partir do momento em que se inicia a venda dos frutos.

$\mathrm{O}$ projeto apresentou um VPL de R\$ $29.010,49$ e B/C maior que 1 , equivalente a 6,16 . Considerando que boa parte das receitas se dá a partir do ano 10, o lucro operacional até esse momento é negativo. A TIR foi de $26,99 \%$, indicando também a viabilidade dessa atividade. A partir do VAE $(\mathrm{R} \$ 3643,01)$, pode se chegar à receita média por hectare de $\mathrm{R} \$ 303,58$. $\mathrm{mês}^{-1}$.

A produção de equilíbrio $(\mathrm{PrE})$ quando se considera apenas a venda da madeira $\left(153,12 \mathrm{~m}^{3}\right)$, indica que os 71,25 $\mathrm{m}^{3}$ de madeira produzidos não serão suficientes para cobrir, sozinho, os custos operacionais totais, sendo necessário o aumento da produtividade em $81,87 \mathrm{~m}^{3}$ ou aumento do PVU. $\mathrm{O}$ preço de equilíbrio da madeira de $\mathrm{R} \$ 46,27$ indica que a agroindústria rural de pequeno porte tem receita líquida negativa quando vende apenas a madeira de $\mathrm{R} \$ 24,73$ a cada $\mathrm{m}^{3}$ comercializado.

Para agregar valor aos plantios, pode-se optar pelo corte da madeira e entrega das toras nas serrarias, conseguindo assim, melhores preços na venda da madeira. A melhor alternativa consiste em, no mínimo, desdobrar as toras em pranchas antes de vendê-las. Porém, deve ser observada a distância do pátio para a entrega, avaliando assim se a distância inviabiliza ou não essa alternativa.

\section{CONCLUSÕES}

Os critérios de avaliação econômica utilizados (VPL, TIR, B/C e VAE) indicaram viabilidade de implantação da agroindústria rural de pequeno porte e estabelecimento de plantios de jatobá.

Contudo, as receitas do projeto devem incluir, além da receita da venda das sementes, a comercialização da polpa ou a remuneração por serviços ambientais. 
TABELA 6: Atividades envolvidas no plantio para um horizonte de 20 anos e fluxos de caixa líquido e acumulado.

TABLE 6: Activities involved in planting for a 20-year horizon and accumulated net cash flows.

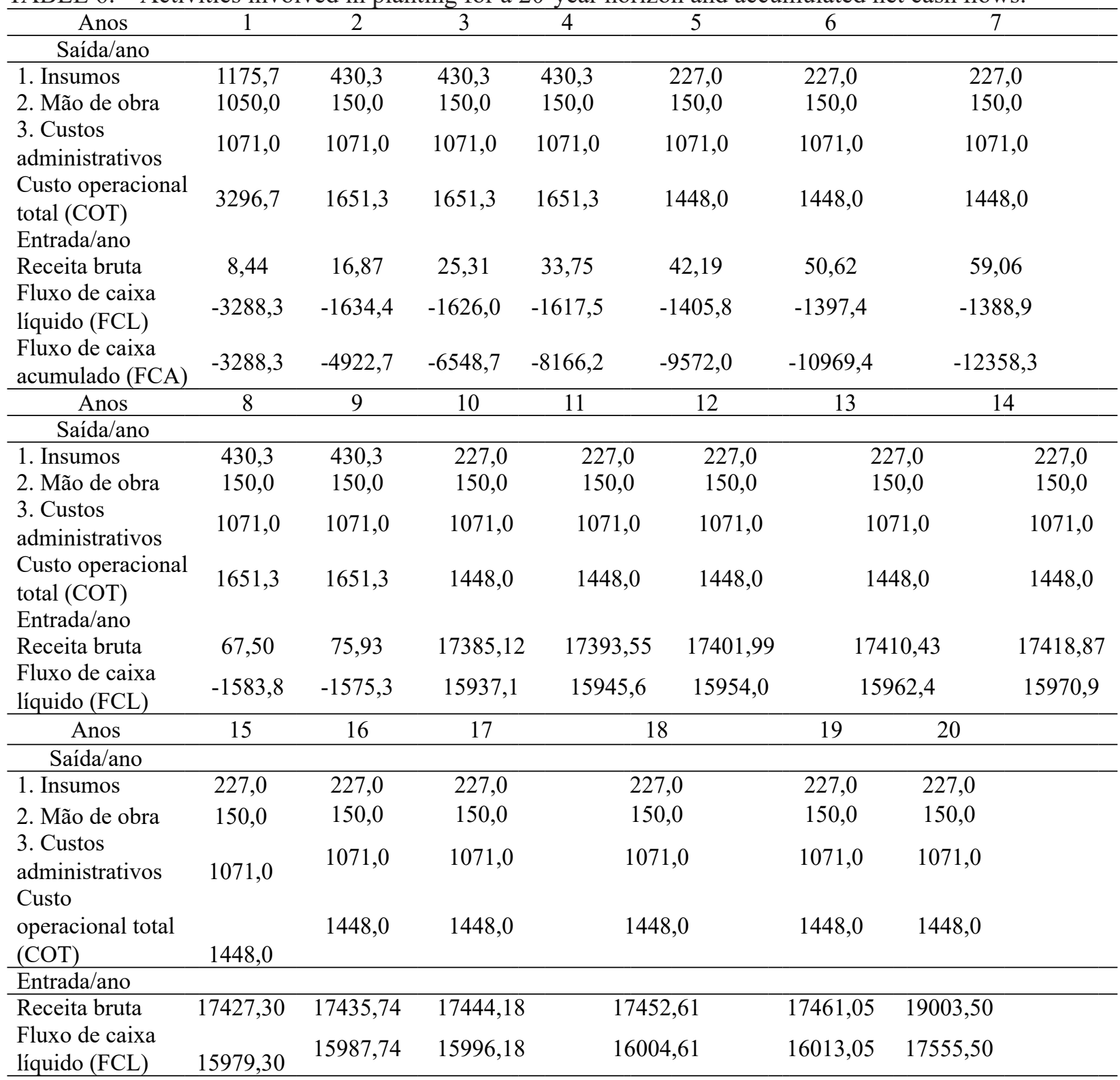

\section{REFERÊNCIAS BIBLIOGRÁFICAS}

ALVES, R. V. Estudo de caso da comercialização dos produtos florestais não madeireiros (PFNM) como subsídio para Restauração Florestal. 2010. 114f. Dissertação (Mestrado em Ciências Florestais) - Universidade Federal de Viçosa, Viçosa, 2010.

ANTEZANA, F. L. Crescimento inicial de $\mathbf{1 5}$ espécies nativas do bioma cerrado sob diferentes condições de adubação e roçagem, em PlanaltinaDF. 2008. 84 p. Dissertação (Mestrado em Ciências Florestais) - Universidade Federal de Brasília,
Brasília, 2008.

ARNOLD, J. E. M. et al. Structure and growth of small enterprises using forest products in southern and eastern Africa. OFI Occasional Paper n. 47. Oxford Forestry Institute, Oxford. 1994.

BALZON, D. R. Avaliação econômica dos produtos florestais não madeiráveis na Área de Proteção Ambiental - APA de Guaratuba - Paraná. 2006, 195p. Tese (Doutorado em Ciências Florestais). Universidade Federal do Paraná, Curitiba, 2006.

BARROS, N. F. et al. NUTRICALC 2.0: 
Sistema para cálculo de balance nutricional y recomendación de fertilizantes para el cultivo de eucalipto. Bosque, v.16, n.1. 1995.

BONFIM, S. L. do. Viabilidade econômicofinanceira de extração sustentada de múltiplos produtos em Floresta Estacional Semidecidua Secundária na microrregião do entorno de Brasília. Dissertação de mestrado. 2010, 187 p. Universidade de Brasília, Brasília, 2010.

BOZZA, A de F. O. Alternativas alimentares com frutos nativos do cerrado. In: JORNAL DA PONTIFÍCIA UNIVERSIDADE CATÓLICA DE GOIÁS. Ano XXIII, n.516. Goiânia, 2012.

CARPANEZZI, A. A. Germinação de sementes de jutaí-açu (Hymenaea courbaril L.) e de jutaí-mirim (H. parvifolia Huber) escarificadas com ácido sulfúrico comercial. Circular Técnica n. 19, Belém, p.17, 1981.

CORRÊA, P. Dicionário de plantas úteis do Brasil - MA. IBDF, 1984.

FERREIRA, C. A. C.; SAMPAIO, P. de T. B.; Jatobá: Hymenaea courbaril. In: CLAY, J. W.; SAMPAIO, P. DE T. B.; CLEMENT, C. R. Biodiversidade Amazônica: exemplos e estratégias de utilização. Manaus, Amazonas. 2000.p.216-225.

FIEDLER, N. C.; SOARES, T. S.; SILVA, G. F. da. Produtos Florestais Não Madeireiros: Importância e Manejo Sustentável da Floresta. Revista Ciências Exatas e Naturais, Guarapuava, v.10 n. 2, p.16, 2008.

GONÇALO, J. E. Gestão e comercialização de Produtos florestais não madeireiros (PFNM) da biodiversidade no Brasil. In:XXVI ENCONTRO NACIONAL DE ENGENHARIA DE PRODUÇÃO - ENEGEP, 2006, Fortaleza.Anais... 2006, 9 pg.

GRAÇA, L. R., RODIGHERI, H. R. CONTO, A. J. Custos florestais de produção: conceituação e aplicação. Documentos, n. 50. Colombo: Embrapa Florestas, 32p. 2000.

LEWIS, G. et al. Legumes of the World. Kew Publishing. 28 ed., 8p., 2005.

LIMA, A. de et al. Manejo da seiva do jatobá (Hymenaea courbaril L.) por famílias tradicionais na Reserva Extrativista Chico Mendes, Acre Brasil. In: VIII CONGRESSO DE ECOLOGIA DO BRASIL, 2007, Caxambu. Anais... Caxambu: MG, $3 p$.

LIMA, D.U.; LOH, W.; BUCKERIDGE, M.S. Xyloglucan-cellulose interaction depends on the side chains and molecular weight of xyloglucan. Plant Physiology and Biochemistry, v. 42, n. 5, pg. 389-394. 2004.
LIMA-NISHIMURA, N. et al. A xyloglucan from seeds of the native Brazilian species Hymenaea courbaril for micropropagation of Marubakaido and Jonagored apples. Cell Biology and Morphogenesis.v.21, p.402-407, 2003.

LISBOA, C. G. S. Possibilidade de uso de polissacarídeos de plantas extraídos de diferentes fontes, uma perspectiva de sustentabilidade. 2008, 254p. Tese (Doutorado em Biologia Celular) - Universidade Estadual de Campinas, Campinas, 2008.

NAUTIYAL, J.C. Forest economics. Principles and applications. Toronto: Canadian Scholars' Press, 1988. 851p.

PEARCE, D. Recuperação Ecológica para Conservação das Florestas e Perspectivas da Economia Ambiental. Conferência IBAMA/ODA/ ICI sobre recuperação ecológica para conservação das florestas. Brasília, 1990.

POINAR JÚNIOR, G.; BROWN, A. E. Hymenaea mexicana sp. (Leguminosae: Caesalpinoideae) from Mexican amber indicates Old World connections. Botanical Journal of the Linnean Society, p.125132, 2002

REZENDE, J.L.P.; OLIVEIRA, A.D. Análise econômica e social de projetos florestais. Viçosa: Universidade Federal Viçosa, 2001. 389p.

SHANLEY, P.; MEDINA, G. Frutíferas e Plantas Úteis na Vida Amazônica. CIFOR, Imazon. Belém, 300p., 2005.

SILVA, M.L.; FONTES, A.A.; LEITE, H.G. Rotação econômica em plantações de eucalipto nãodesbastadas e destinadas a multiprodutos. Revista Árvore, v.23, n.4, p.403-412, 1999.

SILVA FILHO, J. L. V. Análise econômica da produção e transformação em ARPP, dos frutos de Euterpe edulis Mart. Em açaí no município de Garuva, estado de Santa Catarina. 2005, 77p. Dissertação (Mestrado em Agroecossistemas). Universidade Federal de Santa Catarina. Florianópolis, 2005.

SIMEHGO - Sistema de Meteorologia e Hidrologia do Estado de Goiás. Rede Meteorológica da Secretaria de Ciência e Tecnologia do Estado de Goiás. Dados do município de Anicuns, Estação 34. 2012. Disponível em: file://C:/Users/Camila/ AppData/Local/Temp/Rar\$EX00.556/Dados\%20 do\%20 Munic\%C3\%ADpio\%20de\%20Anicuns todo.htm. Acessado: 12 de abril de 2012.

TORRES, M. R. Compilación y análisis sobre los productos forestales no madereros (PFNM) en el Perú. Estudios nacionales sobre productos 
no madereros en América Latina. San Tiago: FAO, 59p., 2001.

WISNIEWSKI, A.; MELO, F. M. de. Borrachas naturais brasileiras. EMBRAPA - CPATU, 59p.
1982.

WRI - WORLD RESOURCES INSTITUTE. The World Bank in the forest sector: a global policy paper. Wasteland News, v. 8, n. 2, p. 6-12, 1990. 